\title{
HGF upregulation contributes to angiogenesis in mice with keratinocyte-specific Smad2 deletion
}

\author{
Kristina E. Hoot, ${ }^{1}$ Masako Oka, ${ }^{2}$ Gangwen Han, ${ }^{2}$ Erwin Bottinger, ${ }^{3}$ \\ Qinghong Zhang, ${ }^{4}$ and Xiao-Jing Wang ${ }^{1,2}$
}

\begin{abstract}
${ }^{1}$ Departments of Cell and Developmental Biology and Otolaryngology, Oregon Health and Science University, Portland, Oregon, USA. ${ }^{2}$ Department of Pathology, University of Colorado Denver, Aurora, Colorado, USA. ${ }^{3}$ Charles R. Bronfman Institute for Personalized Medicine, and Department of Medicine, Mount Sinai School of Medicine, New York, New York, USA. ${ }^{4}$ Department of Dermatology, University of Colorado Denver, Aurora, Colorado, USA.
\end{abstract}

\begin{abstract}
TGF- $\beta$ signaling can promote tumor formation and development or suppress it, depending on the cellular context and tumor stage. A potential target of this dual effect of TGF- $\beta$ is HGF, as TGF- $\beta$ can inhibit or promote its expression, although the mechanisms underlying this are largely unknown. In the present study, we found that mice with keratinocyte-specific deletion of the TGF- $\beta$ signaling mediator Smad2 (referred to herein as K5.Smad2 $2^{-/-}$mice), which have increased susceptibility to squamous cell carcinomas (SCCs), exhibited angiogenesis associated with epithelial overexpression of HGF and endothelial activation of the HGF receptor c-Met. Application of a c-Met inhibitor abrogated angiogenesis, suggesting that HGF overexpression plays a major role in angiogenesis associated with epithelial Smad2 loss. On the $\mathrm{Hg} f$ promoter, Smad2 was mainly associated with transcriptional corepressors, whereas Smad4 was mainly associated with the transcriptional coactivator CREB-binding protein (CBP/p300). Smad2 loss caused increased binding of Smad 4 and CBP/p300 to the $\mathrm{Hgf}$ promoter. Consistent with this, knocking down Smad2 in human keratinocytes caused increased levels of HGF, which were abrogated by concomitant knockdown of Smad3 and Smad4. Importantly, the incidence of HGF-positive human SCC was high in cases with Smad2 loss and lower when Smad4 was also lost. We therefore conclude that Smad2 loss causes HGF upregulation via loss of Smad2-mediated transcriptional repression and enhanced Smad3/4-mediated transactivation. Since Smad2 is often downregulated in human SCCs, our data suggest a therapeutic strategy of blocking HGF/c-Met activation for Smad2-deficient SCCs.
\end{abstract}

\section{Introduction}

TGF- $\beta$ signaling can be tumor promoting or tumor suppressing depending on the cellular context and tumor stage (1), and its signaling mediators, Smads, are involved in these dual effects. TGF- $\beta$ ligand binds to heteromers of TGF- $\beta$ type I and type II receptors (TGF- $\beta$ RI and TGF- $\beta$ RII) to induce TGF- $\beta$ RI-mediated phosphorylation of Smad2 and Smad3. Phosphorylated Smad2 (p-Smad2) and $\mathrm{p}-\mathrm{Smad} 3$ bind the common Smad, Smad4, and the heteromeric complexes translocate into the nucleus to regulate transcription of TGF- $\beta$ target genes (2). Smad3 binds to the Smad-binding element (SBE) of a target gene, resulting in binding of Smad 2 and/or Smad4 to the same SBE to transactivate or repress target genes (3). Smads can recruit transcriptional activators, such as $\mathrm{CBP} / \mathrm{p} 300$, to induce gene expression (2). Smad2 can recruit transcriptional corepressors such as TGF- $\beta$-induced factor homeobox protein (TGIF), which has been shown to bind histone deacetylases (HDACs) leading to gene silencing (4). TGIF binds to Smad 2 and Smad3 in competition with $\mathrm{CBP} / \mathrm{p} 300$, so the ratio of $\mathrm{CBP} / \mathrm{p} 300$ to TGIF in the promoter of a target gene depends on the relative levels of these 2 proteins in each cell type (4), and the same may be true for other transcriptional coregulators. Increasing numbers of studies now show competition between Smad2 and Smad3 or Smad4 for transcriptional regulation (5-7).

Authorship note: Kristina E. Hoot and Masako Oka contributed equally to this work.

Conflict of interest: The authors have declared that no conflict of interest exists. Citation for this article: J Clin Invest. 2010;120(10):3606-3616. doi:10.1172/JCI43304.
In human squamous cell carcinomas (SCCs), Smad2 and Smad4 are frequently downregulated through loss of 1 genetic allele or at the mRNA level $(7,8)$, whereas Smad3 is retained (7). Consistently, loss of even 1 allele of Smad 2 in mice is sufficient to increase susceptibility to skin carcinogenesis $(7,9)$ due, in part, to increased Snailmediated epithelial-to-mesenchymal transition (EMT) (7). Mice lacking epithelial Smad4 develop spontaneous SCCs in the skin and oral cavity $(8,10,11)$, indicative of a dominant tumor suppressor function for Smad4. Although Smad3-null keratinocytes transduced with a v-ras oncogene exhibited increased malignancy when grafted to immunocompromised mouse skin (12), Smad3-knockout mice are resistant to skin chemical carcinogenesis $(9,13)$ due to abrogation of TGF- $\beta 1$-mediated inflammation and gene expression critical for tumor promotion (13). Therefore, $\operatorname{Smad} 3$ appears to mediate both tumor suppression and promotion effects of TGF- $\beta$.

One of the potential TGF- $\beta$ target genes involved in dual effects of TGF- $\beta$ on cancer is HGF. HGF acts as a ligand for the receptor tyrosine kinase, c-Met. HGF and its receptor are often overexpressed in cancer (14). Activation of the c-Met receptor can lead to proliferation, antiapoptotic survival, invasion, migration in tumor epithelia, and angiogenesis in tumor stroma (14). In the latter case, HGF has been shown to be an independent, potent angiogenic factor through stimulation of endothelial cell growth, migration, scatter, and elongation $(14,15)$. Previous studies have shown TGF- $\beta$ can promote angiogenesis and tumor invasion via stimulation of HGF expression $(16,17)$. Conversely, TGF- $\beta$ has also been shown to inhibit HGF transcription, potentially through binding a TGF- $\beta$ inhibitory element located approximately $400 \mathrm{bp}$ upstream of the 
A
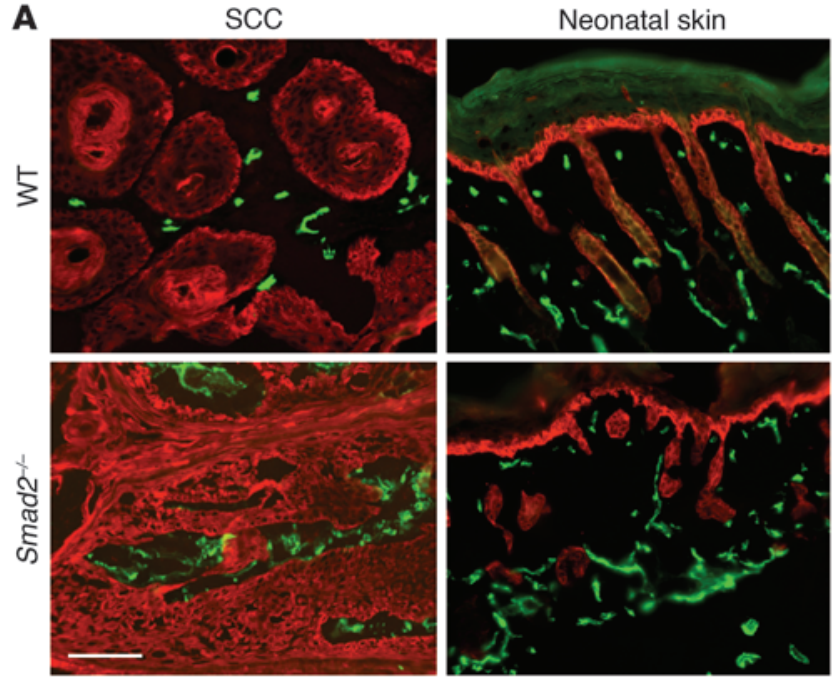

$\mathrm{K} 14 / \mathrm{CD} 31$
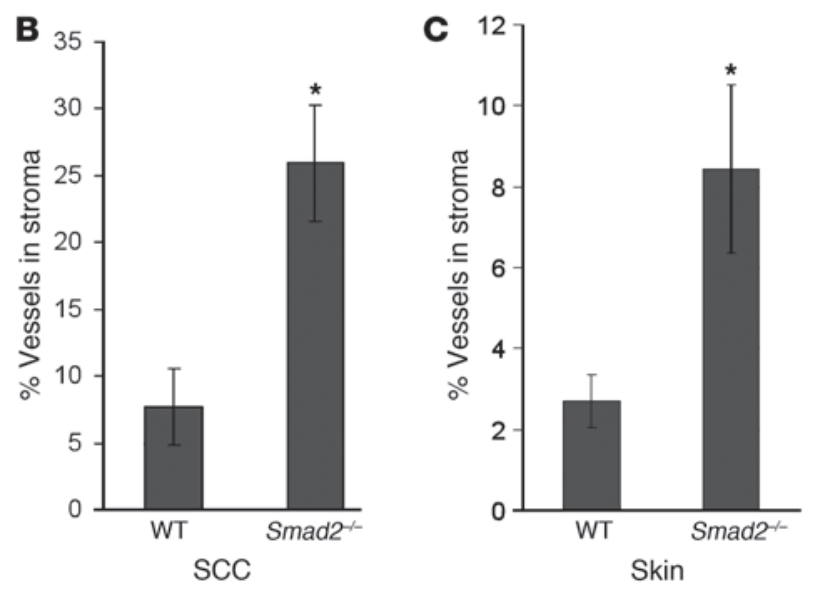

HGF transcription start site $(18,19)$, and abrogation of this effect leads to cancer development (20). The molecular mechanisms and microenvironments controlling the positive or negative effects of TGF- $\beta$ signaling on HGF expression are largely unknown.

In the current study, we found that epithelial Smad2 loss caused increased angiogenesis associated with HGF overexpression and endothelial c-Met activation. Further analysis revealed a repressive role for Smad 2 but an activating role for Smad4 in HGF transcription. Our findings provide important biomarkers for targeted therapy for cancer with Smad2 loss.

\section{Results}

Epithelial Smad2 loss caused increased angiogenesis. Mice with epidermal-specific Smad2 deletion induced at 6 weeks of age were exposed to a 2-stage chemical carcinogenesis protocol as we previously reported (7). We have previously found that keratinocytespecific deletion of Smad2 leads to increased susceptibility to skin carcinogenesis (7). We analyzed angiogenesis in SCCs from the above experiments from 19 mice with keratinocyte-specific deletion of the TGF- $\beta$ signaling mediator Smad2 (referred to herein as K5.Smad2 $2^{-/-}$mice) and 24 WT mice. CD31 staining revealed that K5.Smad2 $2^{-/}$mice demonstrated 3 times the vessel area of control mice $(25.9 \% \pm 4.4 \%$ vs. $7.7 \% \pm 2.9 \%)$ (Figure 1 , A and $\mathrm{B}$ ).

\section{Figure 1}

Increased angiogenesis in K5.Smad2-- SCCs and neonatal skin. (A) Immunofluorescence of K5.Smad2-- SCCs and skin for CD31 (green) showed increased vessel area compared with SCCs derived from WT mice. Keratin 14 (K14, red) was used as a counterstain. Scale bar: $100 \mu \mathrm{m}$. (B) Quantification of percentage of vessel-covered stromal area in K5.Smad2-- SCCs as determined by quantitation of immunofluorescence images. (C) Quantification of percentage of vessel-covered stromal area in $\mathrm{K} 5$. Smad $2^{-/-}$neonatal skin as determined by quantitation of immunofluorescence images. ${ }^{*} P<0.05$. Data are expressed as mean \pm SEM.

To assess whether increased angiogenesis in K5.Smad2-/- SCCs was due to epithelial Smad2 loss or due to the secondary effects of carcinogenesis, we examined angiogenesis in the skin and oral cavity of K5.Smad2 $2^{--}$and WT mice. Smad2 was deleted in the epidermis at birth or in oral epithelia of 5-week-old mice by RU486 application topically or in oral cavity of K5.CrePR1/Smad2f/f bigenic mice as we previously described $(7,8)$. On days $3-5, K 5$. Smad2 $2^{--}$skin and WT skin treated with RU486 were excised for CD31 staining. K5.Smad2 $2^{--}$neonatal skin contained approximately 4 times the stromal area covered in vessels compared with WT neonates $(8.4 \% \pm 2.1 \%$ vs. $2.7 \% \pm 0.7 \%)$ (Figure $1, \mathrm{~A}$ and C). Similar results were also seen in oral tissues (not shown). These results indicate $\operatorname{Smad} 2$ loss in keratinocytes was sufficient to increase angiogenesis in the underlying stroma.

Activated HGF signaling contributed to angiogenesis associated with epithelial Smad2 loss. Since TGF- $\beta$ is a known positive mediator of angiogenesis via endothelial TGF- $\beta$ R Alk1-mediated Smad1/ Smad5 activation (21), we assessed whether K5.Smad2-/- SCCs had increased endothelial TGF- $\beta$ signaling. As previously reported, K5.Smad2-/- SCCs do not have increased TGF- $\beta 1$ ligand when compared with WT SCCs (7). Consistently, K5.Smad2-/- SCCs and skin did not show increased staining of endothelial pSmad1/5/8 compared with WT (Supplemental Figure 1; supplemental material available online with this article; doi:10.1172/JCI43304DS1). These data suggest that angiogenesis in K5.Smad2 $2^{--}$SCCs is not a direct effect of TGF- $\beta$ signaling in tumor stroma.

Since K5.Smad2-/- SCCs had increased angiogenesis independent of TGF- $\beta$-mediated angiogenesis, we screened potential angiogenesis regulators associated with epithelial Smad2 loss, using an angiogenesis microarray from Superarray (OMM-033). Among the angiogenesis factors included in the array (e.g., VEGF, Flt, sFLT, MMPs, MAPKs, FGFs, IL-8, PDGFs, and TNF- $\alpha$ ), only HGF showed a significant increase in K5.Smad2-/- SCCs compared with WT SCCs (Supplemental Figure 2). Increased HGF was primarily located in tumor epithelial cells as visualized by immunohistochemistry (IHC) staining (Supplemental Figure 3). To determine whether increased HGF ligand in K5.Smad2 $2^{-1-}$ tumors activated its signaling, we examined phosphorylation (activation) status of the HGF receptor, $\mathrm{c}-\mathrm{Met}$ ( $\mathrm{p}-\mathrm{c}-\mathrm{Met})$. Immunofluorescence staining showed that K5.Smad2-/- tumors had increased p-c-Met in both tumor epithelia and endothelia compared with stagematched WT tumors (Supplemental Figure 3). Accordingly, downstream mediators of p-c-Met, e.g., p-Akt and eNOS, were activated in both tumor epithelia and endothelia (Supplemental Figure 3). As seen in tumor samples, K5.Smad2-/- neonatal skin had markedly increased HGF compared with WT skin (Figure 2, A and B). IHC showed that HGF staining was strongest in the epidermis, followed by the superficial dermis in $\mathrm{K}^{2} . \mathrm{Smad2}^{-/-}$skin (Figure 2A). 
A
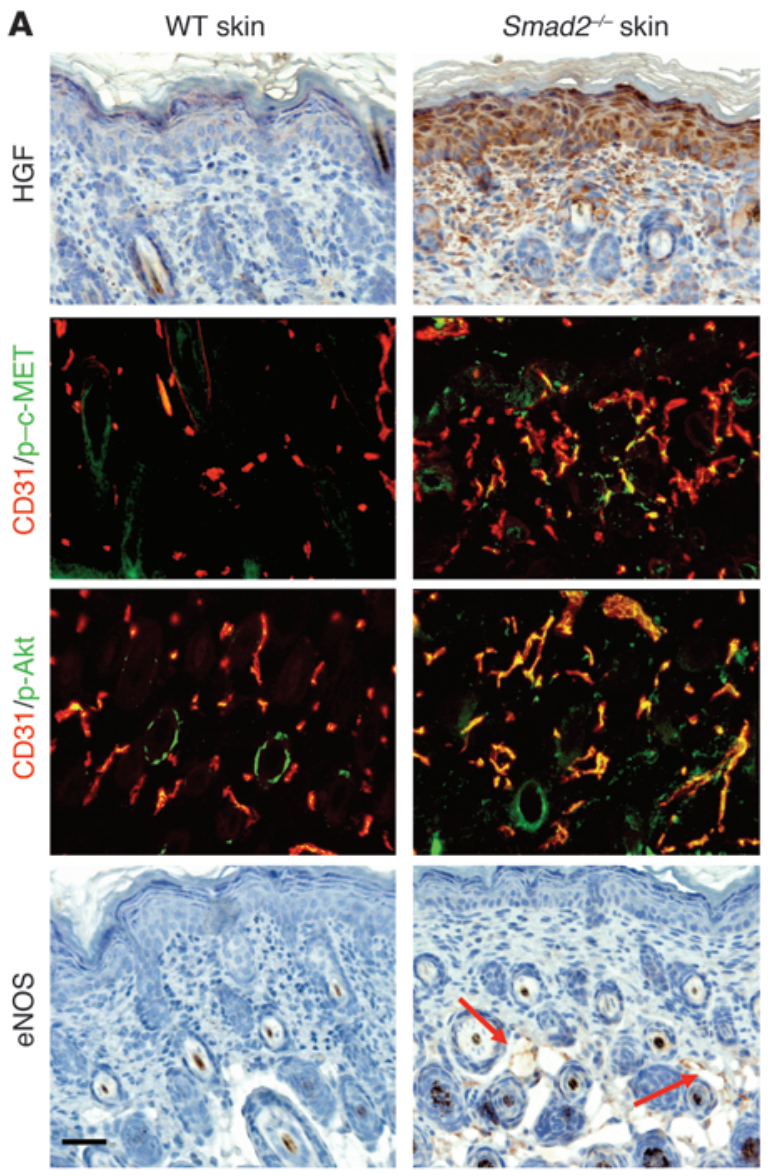

B

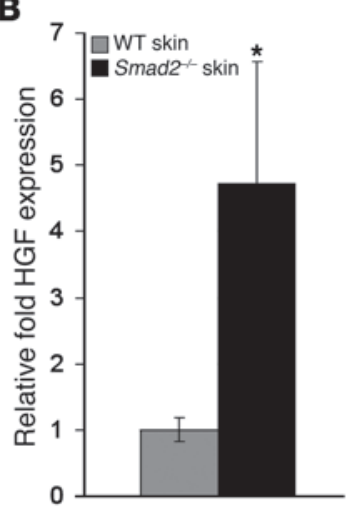

\section{Figure 2}

Increased HGF with activation of its downstream mediators in endothelial cells in $K 5$. Smad2-/- skin. (A) IHC shows increased HGF staining in $\mathrm{K} 5 . \mathrm{Smad}^{-/-}$epidermis and dermis compared with WT skin (first row). Immunofluorescence (IF) shows K5.Smad2 ${ }^{-/}$skin had increased activated endothelial HGF receptor, $p-c-M e t$ (green, second row, CD31 counterstain in red), compared with WT skin. HGF signaling pathway activation demonstrated by increased endothelial p-AKT (green, third row, CD31 counterstain in red) and IHC for eNOS (fourth row) in K5.Smad2 ${ }^{-/}$skin. Arrows indicate endothelial eNOS. Scale bar: $100 \mu \mathrm{m}$. (B) qRT-PCR demonstrating an increased HGF mRNA expression level in K5.Smad2-/- skin compared with WT skin (fold). HGF level in WT skin was arbitrarily assigned as 1.5 skin samples from each group were used. ${ }^{*} P<0.05$. Data are expressed as mean \pm SEM.
This staining pattern suggests keratinocyte-produced HGF acts in a paracrine nature. However, increased p-c-Met and its downstream targets $\mathrm{p}-\mathrm{AKT}$ and $\mathrm{NOS}$ were primarily seen in endothelial cells (Figure 2A), presumably due to a much higher level of c-Met in normal endothelial cells than keratinocytes. These results suggest that HGF upregulation in epithelial cells and its paracrine effect on c-Met activation in endothelial cells is an early effect of epithelial Smad2 loss, whereas activation of c-Met in epithelial cells is secondary to carcinogenesis, presumably due to increased c-Met levels in tumor epithelia compared with normal keratinocytes. We therefore focused on analyzing the direct effect of epithelial Smad2 loss on HGF-induced angiogenesis.

To determine whether HGF upregulation plays a major role in angiogenesis associated with epithelial Smad2 loss, we treated Smad2-deficient skin or oral cavity with the c-Met inhibitor PHA665752 (Tocris). Adult K5.CrePR1/Smad2f/f mice together with WT littermates were treated with RU486 topically in the skin or oral cavity for 5 days to induce Smad2 deletion $\left(\operatorname{Smad} 2^{-/-}\right)$in the epidermis or oral mucosa. Since adult mouse skin has a low level of angiogenesis, we topically treated mice with tetradecanolphorbol-13-acetate (TPA), which induces acute inflammation and angiogenesis. Subsequently, PHA665752 was topically applied to the TPA-treated mouse skin daily for 3 days. WT skin treated with the c-Met inhibitor did not exhibit a significant reduction in vessel density compared with untreated WT skin, indicating that endogenous $\mathrm{HGF} / \mathrm{c}-\mathrm{Met}$ signaling does not significantly contribute to TPA-induced angiogenesis (Figure 3A). However, the vessel density in Smad2-/- skin treated with the c-Met inhibitor was reduced to a level comparable to WT controls (Figure 3A). To determine whether c-Met inhibition also attenuates naturally occurring angiogenesis in tissues with epithelial Smad2 loss, we applied the c-Met inhibitor orally (dissolved in sesame oil) for 3 days. Examination of angiogenesis in oral tissue revealed similar effects of c-Met inhibition on epithelial Smad2 loss-associated angiogenesis in oral tissue (Figure $3 \mathrm{~B}$ and Figure 4). Immunofluorescence staining shows that the c-Met inhibitor did not alter the number of $\mathrm{p}$-Smad2-positive cells in the skin (not shown) or oral mucosa (Figure 4), but significantly reduced $\mathrm{p}-\mathrm{c}-\mathrm{Met}$-positive cells in vessels of $\mathrm{K} 5 . \mathrm{Smad2} \mathbf{2}^{-/-}$stroma (Figure 4). These results suggest that HGF-mediated c-Met activation in endothelial cells is a major factor contributing to angiogenesis associated with epithelial Smad2 loss.

Smad2-mediated transcriptional repression of HGF in keratinocytes. We have previously shown that Smad2 and Smad4 are frequently downregulated while Smad3 is largely retained in human SCCs (7). To determine whether Smad4 loss has an effect similar to Smad2 loss on HGF overexpression, we examined HGF levels in the skin with keratinocyte-specific deletion of Smad4 $(7,8)$. No difference in levels of HGF mRNA (Supplemental Figure 4) and protein (not shown) were found in $\mathrm{K} 5 . \mathrm{Smad4} 4^{-/}$skin, suggesting that Smad4 has little effect on HGF regulation in normal keratinocytes.

Previous reports have shown that HGF is either positively or negatively regulated TGF- $\beta(16,18-20)$. To determine whether Smad2 loss-associated HGF overexpression is linked to Smaddependent TGF- $\beta 1$ effects on HGF regulation, we examined HGF 

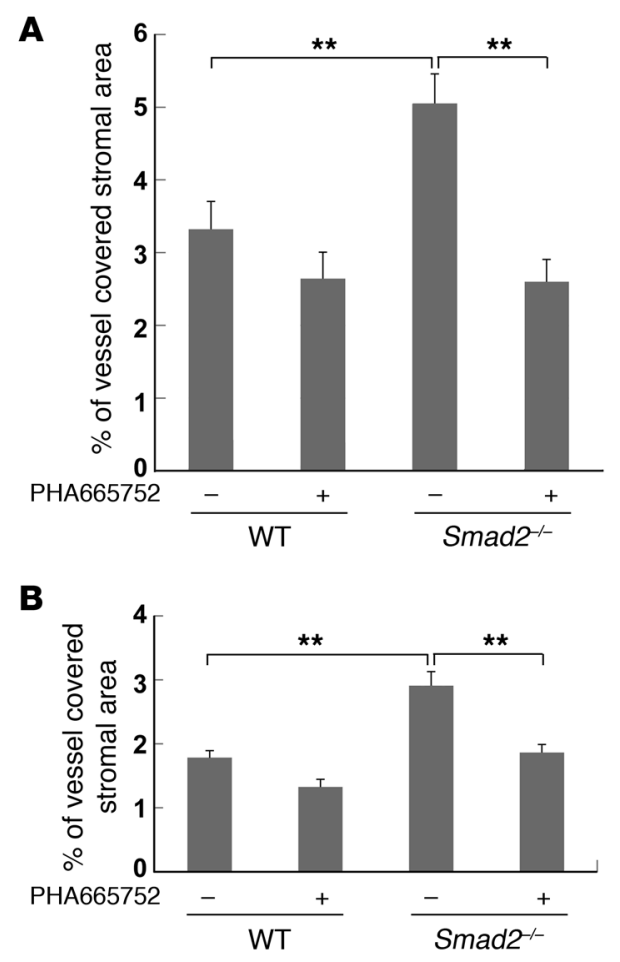

levels after knocking down Smad2, Smad3, or Smad4 in human $\mathrm{HaCaT}$ keratinocytes using individual siRNAs specific for Smad2, -3 , or -4 validated in our previous study, which knocked down each Smad without affecting other Smads (7). We verified knockdown efficiencies at the mRNA and protein levels (Supplemental Figure 5). Similar to previous reports that TGF- $\beta 1$ suppresses HGF expression in normal cells (18-20), $2 \mathrm{ng} / \mathrm{ml}$ TGF- $\beta 1$ treatment of mock-transfected HaCaT keratinocytes for 2 hours caused a significant reduction in HGF expression (Supplemental Figure 6). Similar to the finding in K5.Smad2 $2^{-/-}$skin, knocking down Smad2 resulted in an approximately 4- to 5-fold increase in HGF expression with or without TGF- $\beta 1$ treatment in comparison with corresponding controls (i.e., TGF- $\beta 1$-untreated and -treated $\mathrm{HaCaT}$ cells, respectively) (Supplemental Figure 6). In contrast, knocking down Smad3 or Smad4 had no significant effect on HGF levels with or without TGF- $\beta 1$ treatment (Supplemental Figure 6). These data suggest that $\mathrm{Smad} 2$ loss attenuated endogenous or exogenous TGF- $\beta 1$-mediated HGF inhibition.

The specific link between Smad2 loss and HGF overexpression found in our study prompted us to evaluate whether Smad 2 directly inhibits HGF transcription. From the TRANSFAC database, we identified a SBE -466 bp upstream of the mouse HGF transcriptional start site (TSS), a region previously shown to be repressed by TGF- $\beta$ (18-19). We performed in vivo ChIP assays in neonatal mouse skin using validated Smad antibodies as we previously described (7) and confirmed that $S \operatorname{mad} 2,-3$, and -4 all bound to this site (Figure 5A). To evaluate whether this SBE site is critical for Smad regulation of the HGF promoter activity, we created luciferase reporter constructs with -500 bp HGF promoter sequence with and without an SBE mutation. HaCaT human keratinocytes were transfected with the HGF luciferase constructs, control renilla construct, and individual siRNAs for $\operatorname{Smad} 2,-3$, or -4 . Consistent with the above data, knockdown of Smad2 resulted in a 5 -fold

\section{Figure 3}

Abrogation of angiogenesis by c-Met inhibition in K5.Smad2 ${ }^{-/-}$skin or oral cavity. (A) Quantitation of angiogenesis demonstrates abrogation of angiogenesis by a c-Met inhibitor PHA665752 in TPA-treated K5. Smad2 $2^{--}$skin. (B) Quantitation of angiogenesis demonstrates abrogation of angiogenesis by a c-Met inhibitor PHA665752 in K5.Smad2-/tongue mucosa. ${ }^{* \star} P<0.001$. Data are expressed as mean $\pm \mathrm{SEM}$.

increase in luciferase activity, which was abrogated by mutation of the SBE (Figure 5B). In contrast, knocking down Smad3 or Smad4 did not affect luciferase activity with either WT SBE or mutant SBE (Figure 5B). These results suggest that Smad2 binding to this $\mathrm{SBE}$ is critical for its repressive effect on HGF transcription; hence, Smad2 loss caused HGF overexpression.

We then performed ChIP assays to identify potential corepressors recruited by Smad2 at the -466 bp SBE site. As expected, in K5.Smad2 $2^{-/-}$skin, Smad2 binding was lost (Figure 6A). Conversely, in $\mathrm{K} . \mathrm{Smad4}^{-/-}$skin, Smad2 binding was increased by 8 -fold at the SBE compared with WT skin (Figure 6A). We then performed ChIP assays for corepressors TGIF, CtBP1, and HDACs-1, -2, and -3, which have been shown to be recruited by $\operatorname{Smad} 2$ to other transcriptional targets (2-3). In K5.Smad2-/- skin, TGIF binding to the HGF promoter was significantly reduced whereas CtBP1 and HDAC3 binding was not significantly reduced in comparison with WT skin (Figure 6B), suggesting that TGIF binding to the HGF promoter is primarily recruited by Smad2, whereas CtBP1 and HDAC3 may also be recruited by the remaining Smad4. However, along with increased Smad2 binding in $\mathrm{K}^{2} . \mathrm{Smad4}^{-/-}$skin, TGIF and CtBP1 exhibited modest increases in binding to the SBE of the HGF promoter, whereas HDAC3 binding to this site increased by 45-fold compared with WT skin (Figure 6B). These data indicate Smad2 has a stronger activity than Smad4 to recruit the transcriptional corepressors, particularly HDAC3 to the SBE of the HGF promoter. To confirm that Smad2 directly bound in a complex with HDAC3 on the HGF promoter, we performed a dual-IP ChIP using WT neonatal mouse skin. We immunoprecipitated chromatin using an antibody specific to either Smad2 or Smad4, followed by precipitation of chromatin-Smad complexes using an antibody specific to HDAC3. HDAC3 bound to Smad2 10-fold more than to Smad4 (Figure 6C).

Increased Smad4-mediated transactivation of HGF contributed greatly to HGF overexpression in Smad2-/- keratinocytes. We have previously shown that Smad2 loss in keratinocytes causes increased Smad4 binding and transactivation of the Snail promoter without changes to the level of Smad3 or Smad4 protein expression (7). To assess whether a similar mechanism also contributes to increased HGF overexpression in Smad2-deficient cells, i.e., a compensatory activation of Smad4-mediated HGF transcription, we knocked down Smad2, -3 , or -4 individually or in combination in human $\mathrm{HaCaT}$ keratinocytes and assayed for expression levels of endogenous HGF. Similar to the HGF promoter reporter assay (Figure 5B), siRNA knockdown of Smad2 leads to increased HGF mRNA levels, whereas knocking down either Smad3 or Smad4 alone did not significantly affect HGF expression levels (Figure 7A). However, knockdown of Smad3 and Smad4 together resulted in reduced HGF expression compared with control (Figure 7A). In addition, concomitant knockdown of Smad3 or Smad4 with Smad2 abrogated Smad2 loss-associated HGF overexpression (Figure 7A). These results suggest that endogenous Smad3 and Smad4 
A
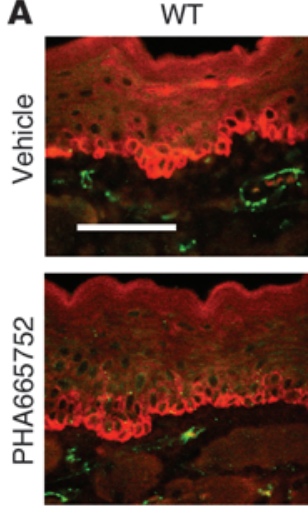

B
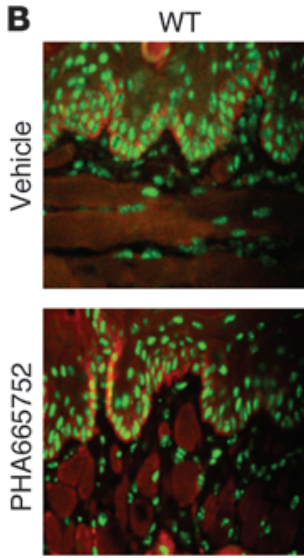

c
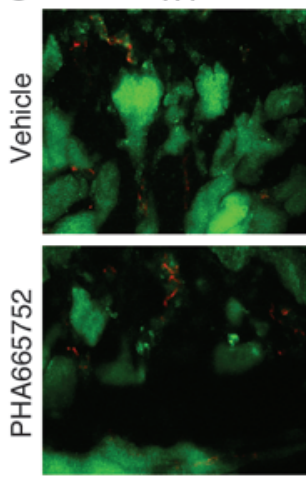

Smad2

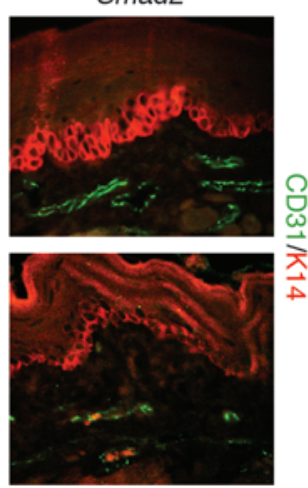

Smad2-

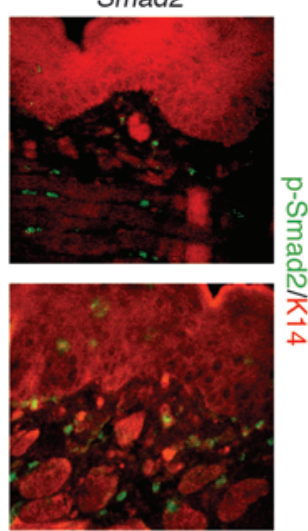

Smad2-

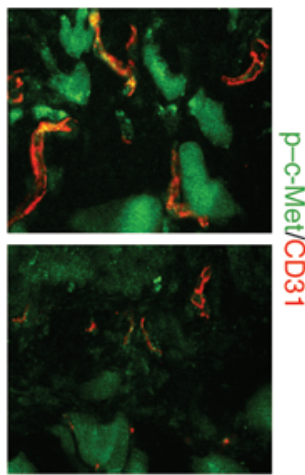

together activate normal HGF expression, which could contribute to HGF overexpression in Smad2-depleted cells.

We then determined whether Smad3 and Smad4 binding to the HGF promoter was increased in K5.Smad2-/- skin. ChIP assays showed that Smad3 binding was modestly increased ( 3.5-fold) and Smad 4 binding was dramatically increased ( $\sim 8$ fold) to the $\mathrm{SBE}$ of the HGF promoter in K5.Smad2 ${ }^{--}$skin (Figure 7B). Since we have shown that Smad2 loss does not cause compensatory Smad4 overexpression (7), increased Smad4 binding to the HGF promoter in Smad2-deficient cells could be target specific instead of global Smad4 activation. To further evaluate whether increased Smad4 binding in $\mathrm{K} 5 . \mathrm{Smad} 2^{-/-}$cells changed the recruitment of transcriptional coactivators on the SBE site of the HGF promoter, we performed ChIP assays for $\mathrm{CBP} / \mathrm{p} 300$, a previously known coactivator of Smad4 (2-3) and RNA polymerase II (RNA Pol II). Consistent

\section{Figure 4}

c-Met inhibition abrogates angiogenesis associated with epithelial Smad2 loss. (A) IF staining of CD31 (green) shows increased angiogenesis in K5.Smad2 $2^{-/-}$tongue mucosa, which was abrogated by a c-Met inhibitor PHA66572. K14 (red) was used for counterstain. (B) IF staining shows that PHA66572 did not affect Smad2 activation, as evidenced by staining of $p$-Smad2 (green). Note that $p$-Smad2 was specifically ablated in tongue epithelial cells but not in $\mathrm{K} 5$. Smad2-/stroma. K14 (red) was used for counterstain. (C) Double IF staining of p-c-Met (green) and CD31 (red) shows that the vessels in K5. Smad2-/- tongue had increased $\mathrm{p}-\mathrm{c}-\mathrm{Met}$ staining (yellow or orange), which was abrogated by PHA66572 treatment. Bright green staining surrounding the vessels represents autofluorescence of the muscles in the tongue. Scale bar: $100 \mu \mathrm{m}$.

with increased Smad4 binding in $K 5 . S m a d 2^{-/-}$skin, CBP/p300 showed increased binding to the SBE of the HGF promoter over 200-fold compared with WT skin (Figure 7D). Additionally, RNA Pol II binding to the SBE of the HGF promoter was increased by 2.5 -fold in $\mathrm{K} 5 . \mathrm{Smad}^{-/-}$skin (Figure 7D). In contrast, in $\mathrm{K} 5 . \mathrm{Smad4}^{-/-}$ skin that has increased Smad2 binding (Figure 6A), CBP/p300 binding was only increased 10 -fold, whereas RNA Pol II binding was actually reduced by 75\% compared with WT skin (Figure 7D), which is consistent with increased binding of Smad2 and corepressors in the HGF promoter (Figure 6). Further, we compared recruitment of CBP/p300 by Smad 2 and Smad4 to the HGF promoter, using a dual-IP ChIP assay in WT mouse skin with an antibody specific for Smad2 or Smad4, followed by immunoprecipitation of Smad/DNA complexes with the CBP/p300 antibody. We found 75 -fold more CBP/p300 bound to Smad4 than to Smad 2 on the HGF promoter (Figure 7E). Taken together, these data show that Smad4 primarily recruits a transcriptional coactivator to the HGF promoter and transactivates HGF; hence, a significant increase in Smad4/CBP binding to the HGF promoter in Smad2-deficient cells contributes greatly to HGF overexpression.

High incidence of HGF overexpression in human SCCs with Smad2 loss. HGF is normally expressed in mesenchymal cells but is often overexpressed in epithelial tissues of developing cancers (22). To determine whether the mechanisms of Smad2 loss-associated HGF overexpression found in our analyses contribute to HGF overexpression in human SCCs, we performed IHC staining of HGF on human SCC tissue arrays containing 74 skin SCCs and 113 head and neck SCCs (HNSCCs) (Biomax). Similar to our previous reports $(7,8)$, approximately $60 \%-70 \%$ of SCCs lost either or both Smad 2 and Smad4 (not shown). HGF was not detectable in normal tissues (Figure 8 ), but was detected in 60\% (45/75) of skin SCCs and in $45 \%$ (38/84) of HNSCCs. Consistent with our findings in animal models and in in vitro analyses, among skin SCCs that lacked Smad2 protein but retained Smad3/Smad4 protein, HGF was detectable in most of the SCCs (Table 1 and Figure 8). HGF-positive cases were reduced in Smad2-negative cases when they also lost Smad4 protein (Figure 8) and were further reduced in SCCs when Smad3 was also lost (Table 1). These data further support that Smad2 loss together with Smad3/Smad4-mediated transactivation contributed to HGF overexpression in at least some human SCC cases. HGF overexpression in cases of all $3 \mathrm{Smads}$ positive or all $3 \mathrm{Smads}$ negative for Smad2, Smad3, and Smad4 could represent Smad-independent mechanisms of HGF regulation. For instance, hypoxia-induced factors and increased MMP activity, which are commonly associated with cancer, largely contribute to HGF induction (22). 
A
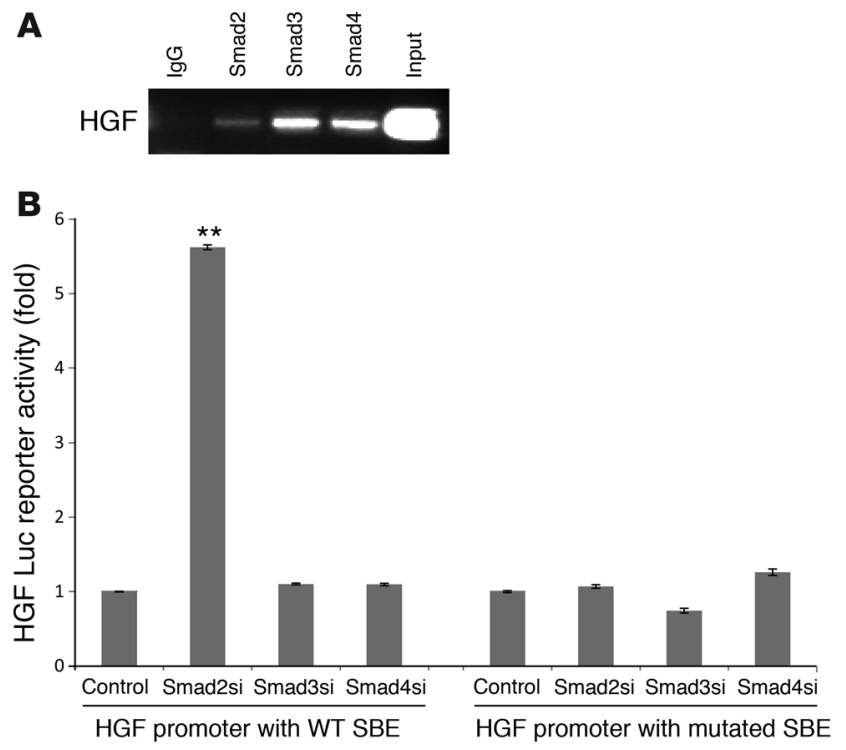

\section{Discussion}

HGF overexpression plays a critical role in angiogenesis associated with epithelial Smad2 loss. We have previously shown that Smad2 loss in keratinocytes caused an early onset EMT, which contributes to increased susceptibility to skin cancer formation and malignant progression (7). In the current study, we observed increased angiogenesis in K5.Smad2-/- tissues and tumors. Since Smad2 was only deleted in keratinocytes, increased angiogenesis in the stroma suggests that angiogenic regulators secreted from keratinocytes were altered by Smad 2 deletion. As loss of a TGF- $\beta$ signaling component in keratinocytes often causes compensatory TGF- $\beta 1$ over-

\section{Figure 5}

Smad2 binding to the SBE of the HGF promoter is critical for transcriptional regulation of HGF. (A) ChIP assay showing Smad2, Smad3, and Smad4 all bound to the SBE located 466 bp upstream of the transcription start site of the HGF promoter. IgG (rabbit) was used as a negative control. (B) siRNA knockdown of Smad2 leads to 5-fold increase in HGF promoter activity, which is abrogated by mutation of the SBE. Knockdown of Smad3 and Smad4 do not increase HGF promoter activity. ${ }^{* *} P<0.001$. Data are expressed as mean \pm SEM.

expression $(8,23)$, we determined whether increased angiogenesis in $K 5 . S m a d 2^{-1-}$ tissues was due to increased TGF- $\beta 1$ that could directly induce angiogenesis (21) or due to increased VEGF, which can be activated by Smad3 and is seen after Smad4 is knocked out in keratinocytes (24) or in breast cancer cells after knocking down Smad2 (25). However, we observed neither increased TGF- $\beta 1$ nor increased VEGF production in nonneoplastic K5.Smad2-/- tissues or SCCs compared with WT samples, possibly due to a lack of further enhancement of Smad3 activation seen in $\operatorname{Smad} 4^{-/-}$keratinocytes (8), which directly transactivates $\operatorname{VEGF}(26,27)$. These results highlight the context-specific nature of Smad transcriptional regulation. Using an unbiased screening, we identified that Smad2 loss induces overexpression of HGF. In nonneoplastic K5.Smad2-/- tissues, we did not observe consequent activation of HGF receptor c-Met in epithelial cells, presumably due to a low level of c-Met in normal epithelial cells. However, at this stage, overexpressed HGF is sufficient to activate c-Met in endothelial cells. Further, treating K5.Smad2 $2^{-1-}$ mice with a c-Met inhibitor completely abrogated increased angiogenesis to a baseline level seen in normal tissues, suggesting that HGF overexpression is a major contributor to angiogenesis associated with epithelial Smad2 loss. This finding has an important implication for a therapeutic strategy targeting SCCs. We have shown that loss of $1 \mathrm{Smad} 2$ allele, which

\section{Figure 6}

Smad2-mediated repression of HGF transcription. (A) HGF promoter occupancy by Smad2 in WT skin, $K 5$. Smad2/-- skin, and K5.Smad4/- skin. Smad2 ${ }^{-/-}$skin has reduced Smad2 binding with remainder attributable to nonepithelial tissues. Smad4 ${ }^{-/}$skin showed an 8-fold increase in Smad2 binding. (B) Transcriptional repressors TGIF, CtBP1, and HDAC3 bound to the HGF promoter with greater affinity in $\mathrm{K5} . \mathrm{Smad}^{-/-}$and reduced affinity in $\mathrm{K} 5 . \mathrm{Smad2^{-/- }}$ skin compared with WT skin by comparative ChIP. (C) Dual-IP ChIP was performed by first immunoprecipitating Smad2 and Smad4 on the HGF promoter in WT skin, then performing a second IP to HDAC3. ${ }^{*} P<0.05 ;{ }^{*} P<0.01$. Data are expressed as mean \pm SEM.
A
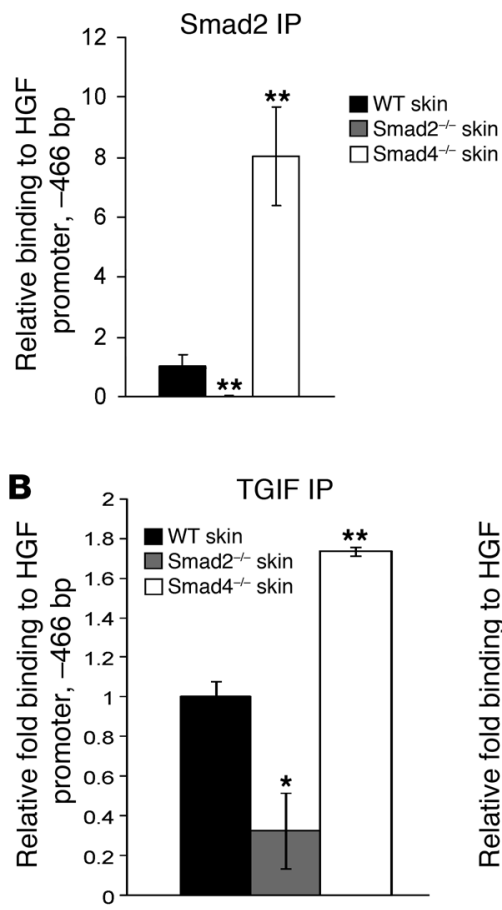

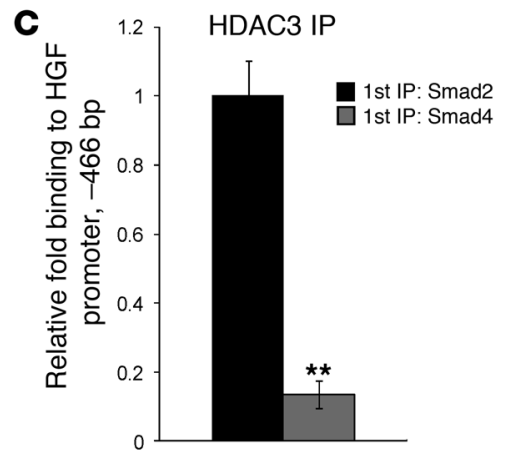

CtBP1 IP

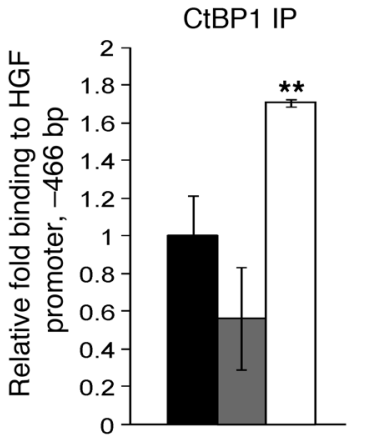

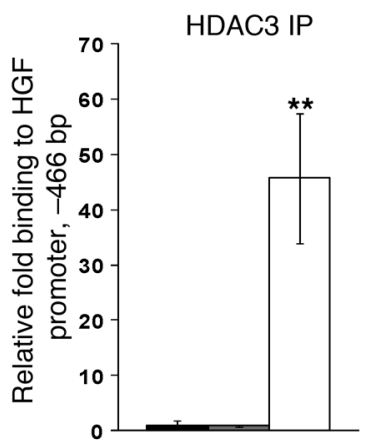


A
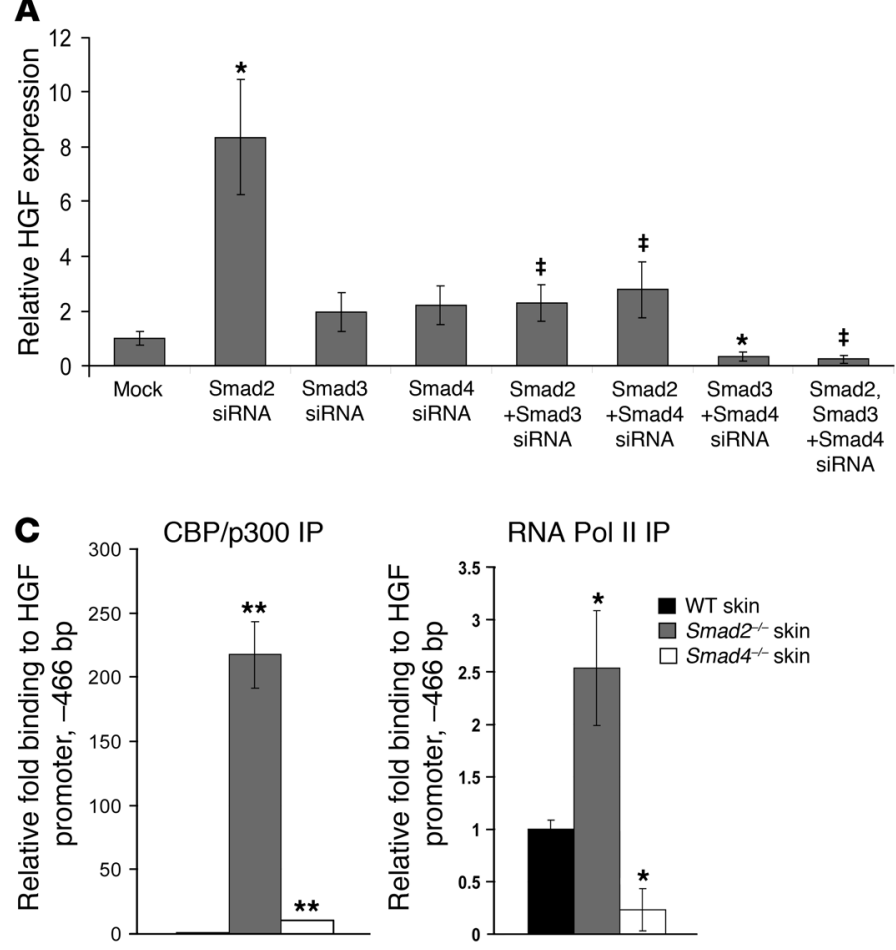

B
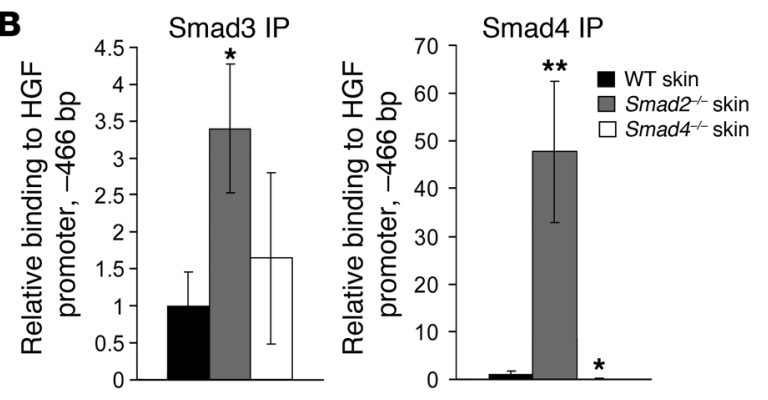

Figure 7

Smad4-mediated transactivation of HGF transcription. (A) Knockdown of Smad2 dramatically upregulates HGF expression, which is abrogated by concomitant knockdown of either Smad3 or Smad4. Knockdown of Smad3 and Smad4 leads to reduced HGF expression compared with mock treatment. ${ }^{*} P<0.05$ compared with mock treatment. ${ }^{\ddagger} P<0.05$ compared with Smad2 siRNA. (B) HGF promoter occupancy by Smad3 and Smad4 in WT skin, K5.Smad2/- skin, and K5.Smad4-/- skin. As expected, Smad4 binding is reduced in Smad4-/- skin. Smad2/- skin showed an increase in Smad3 and Smad4 binding. ${ }^{*} P<0.05$ compared with WT skin. (C) Transcriptional activators CBP/p300 and RNA Pol II bound with greater affinity in $\mathrm{K}_{5} . \mathrm{Smad2}^{--}$skin, correlating with Smad2 loss-associated HGF upregulation. CBP/p300 binding was modestly increased in Smad4-/ skin, which showed an increase in Smad2 binding. RNA Pol II was reduced in Smad4-/- skin. ${ }^{*} P<0.05 ;{ }^{* *} P<0.01$ compared with WT skin. (D) Dual-IP ChIP was performed by immunoprecipitating Smad2 and Smad4 on the HGF promoter in WT skin, then performing a second IP to $\mathrm{CBP} / \mathrm{p} 300$. Transcriptional activator $\mathrm{CBP} / \mathrm{p} 300$ bound to Smad4 with 75 -fold greater affinity than to $\mathrm{Smad2}$. ${ }^{*} P<0.05$. Data are expressed as mean $\pm \mathrm{SEM}$.

contributes to at least a $50 \%$ reduction of Smad2 protein, occurs in approximately $40 \%$ of human SCCs and that overall Smad 2 protein loss occurs in approximately $70 \%$ of human SCCs (7). Our current study suggests that Smad2 loss is an important factor contributing to HGF overexpression in human SCCs. Since Smad2 is haploid insufficient, i.e., $50 \%$ of Smad2 loss is sufficient to increase skin cancer susceptibility (7), it would be difficult to restore genetically lost Smad2 to a normal level when treating SCC patients. Therefore, if we can block Smad2 loss-mediated angiogenesis using a c-Met inhibitor, Smad2 loss-associated malignant progression may be attenuated or delayed. As seen in our current study, since HGF is barely detectable in normal tissue, the c-Met inhibitor did not significantly affect normal angiogenesis, which could be beneficial as a targeted therapy. Nevertheless, since cancer-associated angiogenesis involves multiple pathways and often harbors oncogene addiction (28), it remains to be determined whether blocking HGF-mediated angiogenesis can significantly slow down or starve tumor cells in Smad2-deficient SCCs.

HGF transcription is negatively regulated by Smad2 but positively regulated by Smad4. TGF- $\beta$ can stimulate HGF production $(16,17,29)$ but can also represses HGF $(30,31)$. As summarized in Figure 9 , our current study revealed an important mechanism underlying this context-specific effect of TGF- $\beta$ signaling on HGF transcriptional regulation, which largely depends on the ratio of Smad2 and Smad 4 in cells. In normal keratinocytes, Smad2, -3 , and -4 all bind to the $-466 \mathrm{bp}$ SBE of the HGF promoter (Figure 5). In this complex, Smad2 primarily recruits transcriptional corepressors (e.g., TGIF, CtBP1, and HDAC3; Figure 6), whereas Smad4 primarily recruits transcriptional coactivator CBP/p300 (Figure 7). Since normal keratinocytes produce very low levels of TGF- $\beta$, the recruitment of either corepressors or coactivators are expected to be at low levels. Together with the balance between the recruitment of corepressors and coactivators, virtually no HGF can be detected in normal keratinocytes. Because Smad3 has the strongest DNA binding, loss of Smad 2 only modestly increased Smad 3 binding, whereas loss of Smad4 did not significantly affect Smad 3 binding to the HGF promoter. Smad4 loss in normal keratinocytes had no significant effect on baseline HGF expression, despite increased binding of Smad2 and corepressors, i.e., TGIF, CtBP1, and HDAC3. Two possibilities could contribute to this result. First, consistent with a previous report that Smad2 can recruit both corepressors and coactivator CBP/p300 (4), increased CBP/p300 binding to the HGF promoter was also seen in $\mathrm{K} 5 . \mathrm{Smad} 4^{-/-}$skin, which was presumably a result of increased Smad2 binding. Second, the remaining 

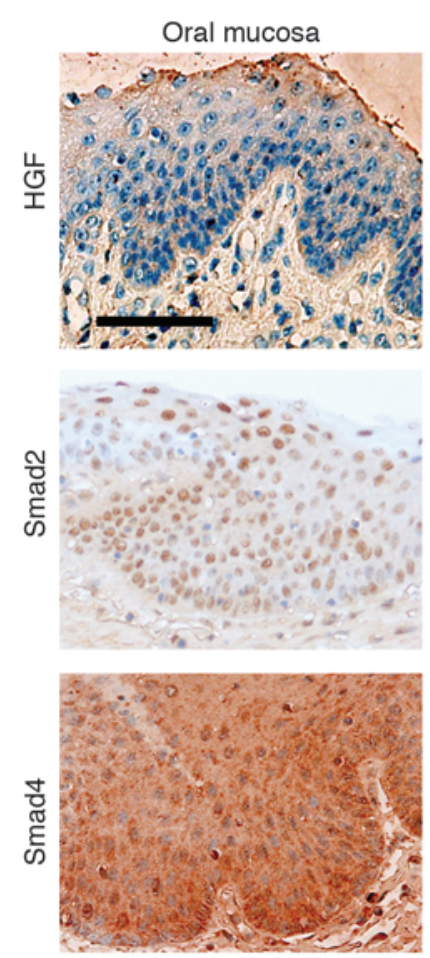
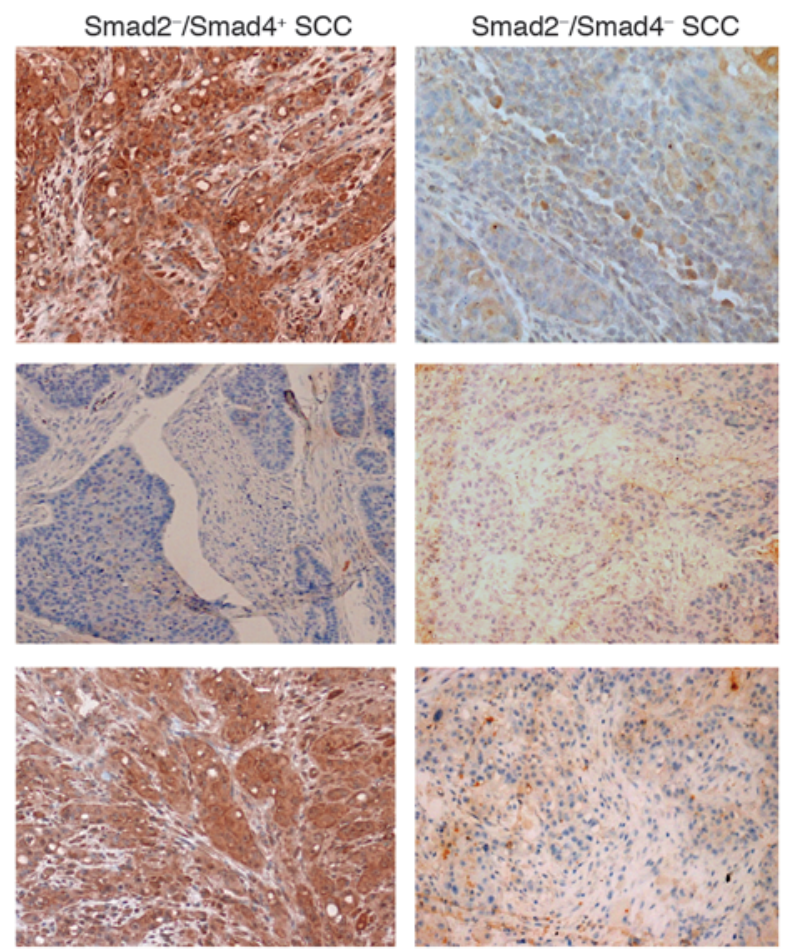

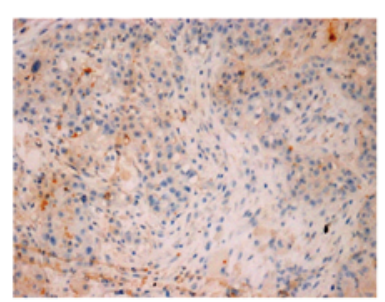

\section{Figure 8}

HGF expression correlates with Smad2 loss but Smad4 retention in human SCCs. $\mathrm{IHC}$ staining showing that normal oral mucosa is HGF negative but positive for both Smad2 and Smad4. In SCCs from head and neck (here) or skin (not shown), epithelial HGF staining correlated with the Smad2-negative, Smad4-positive staining pattern. In SCC without strong staining of both Smad2 and Smad4, HGF staining in epithelia was weak. Scale bar: $100 \mu \mathrm{m}$.
Smad3 could still transactivate HGF, as knocking down both Smad3 and Smad4 exhibited reduced baseline HGF levels. Therefore, the balance between Smad2/Smad3-mediated transactivation and an increase in Smad2-mediated repression kept HGF levels largely unaffected in Smad4 $4^{-/}$keratinocytes. In contrast to Smad4 loss, Smad2 loss had a dramatic effect on HGF levels. Smad2 loss caused a dramatic increase in Smad4 binding to the HGF promoter. The shift from Smad 2 binding to Smad4 binding also caused a significant increase in the recruitment of transcriptional coactivator $\mathrm{CBP} / \mathrm{p} 300$. Therefore, the significant increase in $\mathrm{CBP} / \mathrm{p} 300$ binding apparently dominates transactivation of HGF. Supporting this notion, ablating Smad2 in keratinocytes resulted in an increased HGF expression, which was abrogated when Smad4 was also ablated. The correlation between HGF expression and Smad2-negative/ Smad4-positive status in human SCCs is also consistent with our molecular analysis and our findings from $\mathrm{K} 5 . \mathrm{Smad2} 2^{--}$and $\mathrm{K} 5$. $S$ mad4 $4^{-/}$mouse models. In addition to the direct transcriptional regulation of HGF by Smad2 and Smad4, it has been shown that mesenchymal cells have HGF-dependent angiogenesis (30-34). Since Smad2 $2^{-/-}$keratinocytes undergo EMT (7), this may also allow a mesenchymal transcriptional environment that promotes HGF transcription.

In summary, we report that Smad2 normally represses HGF expression, and its loss caused HGF overexpression associated with loss of this repression and, perhaps even more, with increased Smad4 transactivation of HGF. Increased HGF contributes greatly to Smad2 loss-associated angiogenesis, which can be abrogated by inhibition of c-Met. Our study indicates that although Smad4 is a potent tumor suppressor, Smad2 loss-associated increase in Smad4 binding to the HGF promoter beyond a physiological level facilitates angiogenesis, which could contribute to tumor promotion. Our study necessitates future analysis of whether HGF-mediated angiogenesis con- tributes to tumor formation and malignant progression in tissues with epithelial Smad2 loss and therefore can be pharmacologically targeted for SCC treatment.

\section{Methods}

All studies were reviewed and approved by IACUC and IRB at Oregon Health and Science University and by IACUC and IRB at University of Colorado Denver.

Generation of inducible and keratinocyte-specific Smad2-and Smad4-knockout mice. Inducible $\mathrm{K} 5 . \mathrm{Smad2}^{-\gamma^{-}}$and $\mathrm{K} 5 . \mathrm{Smad4} 4^{-/-}$mice were generated as we previously reported $(7,8)$. Cre-mediated $S m a d 2$ or $S m a d 4$ deletion in keratinocytes was achieved with topical application of RU486 $(20 \mu \mathrm{g}$ in $100 \mu \mathrm{l}$ ethanol in the skin or $40 \mu \mathrm{g}$ in $50 \mu \mathrm{l}$ in sesame oil in oral mucosa) once a day for 3-5 days at time points specified in the Results section. Smad2 or Smad4 gene deletion was detected by PCR on DNA extracted from RU486treated skin, using deletion-specific primers $(35,36)$.

RNA extraction and quantitative RT-PCR. Total RNA was isolated from mouse skin and tumors using RNAzol B (Tel-Test), as previously described (37), and further purified using a QIAGEN RNeasy Mini Kit (QIAGEN).

\section{Table 1}

HGF-positive cases correlated with Smad2-negative/Smad4-positive status

\section{Smad status}

Smad2-Smad4+Smad3+ Smad2-Smad4-Smad3+ Smad2+Smad4-Smad3+ Smad2-Smad3-Smad4Smad2+Smad3+Smad4+

\section{HGF-positive cases/ total skin SCC cases}

$8 / 8(100 \%)$
$27 / 48(56 \%)^{B}$
$2 / 3(67 \%)$
$0 / 1(0 \%)$
$8 / 15(56 \%)^{A}$

\section{HGF-positive cases/} total HNSCC cases

\section{$11 / 13(85 \%)$}

$17 / 39(44 \%)^{\mathrm{B}}$ $6 / 20(35 \%)^{\mathrm{B}}$ $2 / 9(22 \%)^{B}$ $2 / 3(66 \%)$ 

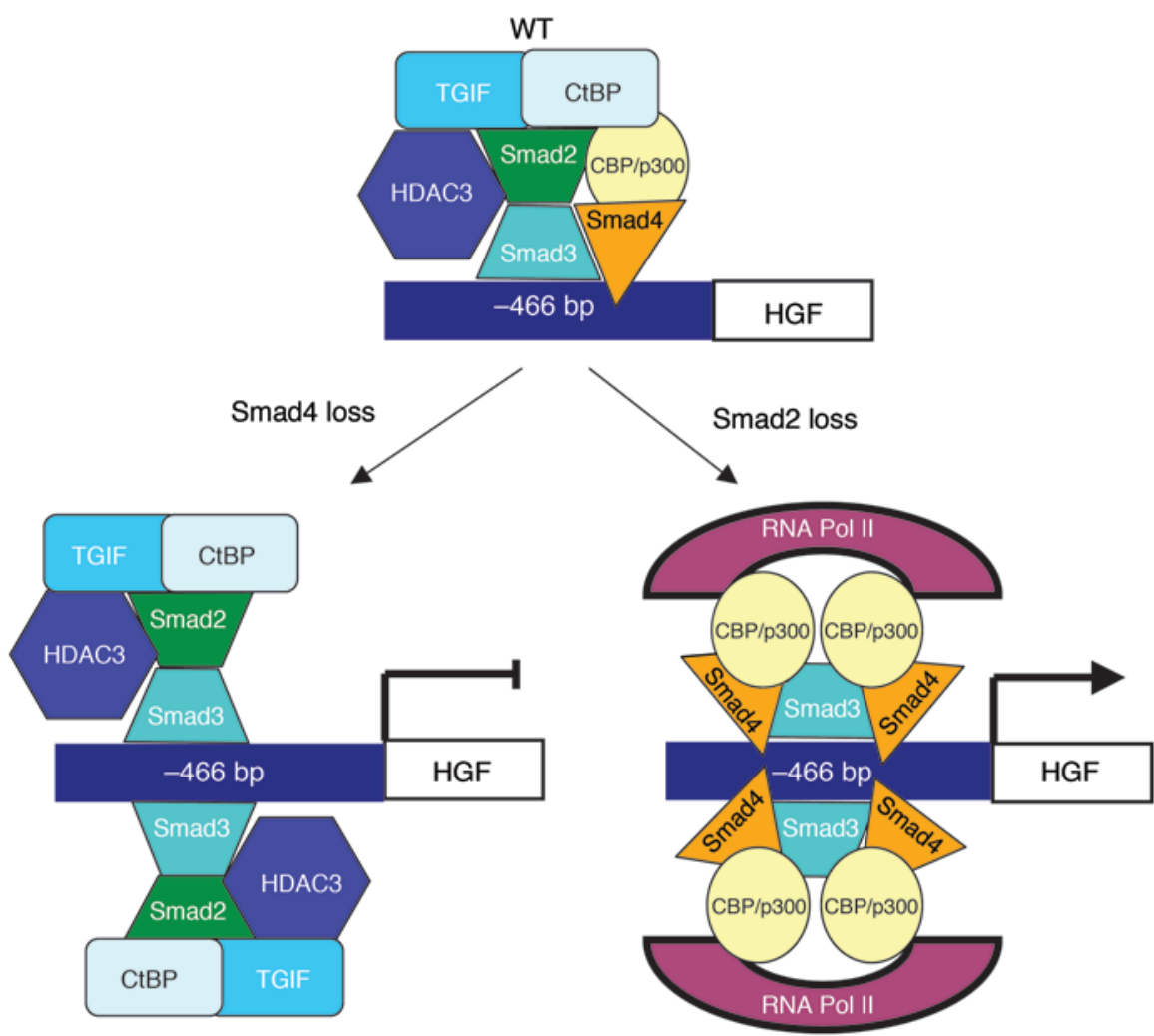

\section{Figure 9}

Schematic of Smad transcriptional regulation of HGF. WT skin has Smad2, -3, and -4 bound to the HGF promoter 466 bp upstream of the TSS, with the various Smads having recruited transcriptional activators (CBP/p300) and repressors (TGIF, CtBP1, and HDAC3). When Smad4 is lost, increased Smad2 is bound, recruiting transcriptional repressors and preventing HGF expression. In contrast, when Smad2 is lost, increased Smad3 and Smad4 bind to the HGF promoter, recruiting transcriptional activators and promoting HGF expression.
Quantitative RT-PCR (qRT-PCR) was performed as we have previously described (38). HGF levels were determined using Power SYBR Green Master Mix (Applied Biosystems) and custom primers (for mouse: 1FGAACTGCAAGCATGATGTGG, 1R-GATGCTGGAAATAGGGCAGAA; for human: 1F- AAAGGACTTCCATTCACTTGC, 1R-CGCTCTCCCTTACTCAAGCTA). A GAPDH RNA probe was used as an internal control. Three to 9 samples from each genotype of mice were used for qRT-PCR. In analyzing the relative expression levels of individual genes, the average expression level from WT samples (unless otherwise specified) of each particular gene being analyzed was set as "1" arbitrary unit.

Tissue histology and IHC. Dissected mouse tissue and tumor samples were fixed in $10 \%$ neutral-buffered formalin at $4^{\circ} \mathrm{C}$ overnight, embedded in paraffin, and sectioned to $6-\mu \mathrm{m}$ thickness. Human tissue arrays of skin SCCs and head and neck SCCs (without personal information) were purchased from Biomax. IHC was performed on paraffin sections of mouse and human SCC samples, as we have previously described (39), using primary antibodies against HGF (1:10; R\&D Systems), p-c-Met (1:50; Cell Signaling), p-Smad2 (1:100; Cell Signaling), Smad2 (1:100; Zymed), Smad4 (1:100; Upstate), and eNOS (1:50; Abcam). Sections were counterstained with hematoxylin. A double-blind evaluation for IHC was performed by 2 investigators using methods we have previously described (39).

Double-stain immunofluorescence. Each section was incubated overnight at $4^{\circ} \mathrm{C}$ with a primary antibody together with either a guinea pig antiserum against mouse K14 (1:400; Fitzgerald), which highlights the epithelial compartment of the skin or oral mucosa (37), or CD31 (1:200; BD Biosciences) which highlights endothelium. The primary antibodies included p-Smad1/5/8 (1:50; Cell Signaling), p-Smad2 (1:100, Cell Signaling), p-AKT (1:100; Cell Signaling), and p-c-Met (1:50; Cell Signaling). Frozen sections were fixed for 2 minutes in methanol. An Alexa Fluor 488-conjugated (green) secondary antibody against the species of the primary antibody (1:400; Molecular Probes) and Alexa Fluor 594-conjugated (red) anti-guinea pig secondary antibody (1:400; Molecular Probes) for K14 counterstain or Alexa Fluor 594-conjugated (red) anti-rat secondary antibody (1:400; Molecular Probes) for CD31 was used to visualize the staining. Sections were visualized under a Nikon Eclipse E600W fluorescence microscope. Images were acquired using MetaMorph (Universal Imaging Corp.) and processed using Adobe Photoshop 6.0. Quantitation of angiogenesis was done using ImageJ software (NIH) and expressed as percentage of stroma occupied by vessels \pm SEM.

c-Met inhibitor treatment. Adult K5.CrePR1/Smad2f/f mice together with their WT littermates were topically treated with RU486 for 5 days to induce Smad2 deletion $\left(\operatorname{Smad2}^{--}\right)$in the skin. These mice were then topically treated with $5 \mu$ g of tetradecanol-phorbol-13-acetate (TPA), which induces acute inflammation and angiogenesis in the skin. Subsequently, a c-Met inhibitor, PHA665752 (Tocris, dissolved in DMSO and then diluted in 100\% ethanol), was topically applied to the skin of the above mice, $16.5 \mu \mathrm{g} /$ mouse, daily for 3 days, beginning on the same day of TPA application. In a separate experiment, Smad 2 was deleted by oral RU486 treatment for 5 days, followed by a c-Met inhibitor PHA665752 (dissolved in sesame oil) daily for 3 days to assess its effect on naturally occurring angiogenesis in oral mucosa.

Quantitative chromatin immunoprecipitation. At least 4 mouse backskins from each group of WT, $\mathrm{K} 5 . \mathrm{Smad2}^{-/-}$, and $\mathrm{K} 5 . \mathrm{Smad4}^{-/-}$mice were homogenized on ice in $5 \mathrm{ml}$ of $1 \%$ formalin and incubated at room temperature for 30 minutes after adding an additional $5 \mathrm{ml}$ of $1 \%$ formalin to each tube. Each sample was then diluted in $1 \mathrm{ml}$ of $10 \times$ Glycine Stop Solution (Active Motif), incubated at room temperature for 5 minutes, and then centrifuged at $250 \mathrm{~g}$ for 10 minutes at $4{ }^{\circ} \mathrm{C}$. The resulting pellet was used for ChIP Enzymatic Digestion following the manufacturer's protocol (Active Motif). Antibodies, $3 \mu$ g each, to Smad2 (Zymed), Smad3 (Upstate), Smad4 (Upstate), RNA Pol II (Upstate), CBP/p300 (Upstate), TGIF (Santa Cruz Biotechnology Inc.), CtBP1 (Upstate), and HDAC3 (Abcam) were used to immunoprecipitate the sheared chromatin complexes. Rabbit IgG 
( $3 \mu \mathrm{g}$; Santa Cruz Biotechnology Inc.) or mouse IgG ( $3 \mu \mathrm{g}$; Upstate) was employed as a negative control for antibody specificity. DNA obtained from eluted beads was used for quantitative PCR using Power SYBR Green Master Mix (Applied Biosystems). Primers encompassing the SBE sites of the HGF promoter (FP, AGTCCAACGGGTCTCAAGTG; RP, CCAAACCACTGCAAAAGGAT) were used for PCR. Positive binding was defined as antibody binding greater than 10 -fold of the IgG-negative control. PCR encompassing the coding region was used as a negative control for specificity of SBE binding. $\Delta \mathrm{Ct}$ values were obtained by normalizing IP Ct values to input values for each group. $\Delta \Delta \mathrm{Ct}$ values were obtained by comparing the $\Delta \mathrm{Ct}$ values of $\mathrm{K} 5 . \mathrm{Smad2}^{-{ }^{--}}$and $\mathrm{K5}$.Smad4 ${ }^{--}$skin to WT skin. Values are expressed as fold change based on $\Delta \Delta \mathrm{Ct}$ values.

Dual-IP ChIP. At least 4 mouse backskins from WT mice were homogenized on ice in $5 \mathrm{ml}$ of $1 \%$ formalin in $10 \mathrm{mM}$ dimethyl adipimidate $2 \mathrm{HCl}$. After homogenizing, another $5 \mathrm{ml}$ of cross-linking solution was added and samples were incubated at $37^{\circ} \mathrm{C}$ for 10 minutes and then at room temperature for 20 minutes. Each sample was then diluted in $1 \mathrm{ml}$ of $10 \times$ Glycine Stop Solution (Active Motif), incubated at room temperature for 5 minutes, and then centrifuged at $250 \mathrm{~g}$ for 10 minutes at $4^{\circ} \mathrm{C}$. The resulting pellet was used for ChIP Enzymatic Digestion following the manufacturer's protocol (Active Motif). Antibodies, $3 \mu$ g each, to Smad2 (Zymed) and Smad4 (Upstate) were used for the initial immunoprecipitation of the sheared chromatin complexes overnight at $4^{\circ} \mathrm{C}$. Rabbit IgG ( $3 \mu \mathrm{g}$, Santa Cruz Biotechnology Inc.) was used as a negative control for antibody specificity. Complexes were eluted from Protein G Beads using $50 \mu$ of the provided elution buffer (Active Motif). Antibodies, $3 \mu$ g each, to CBP/p300 (Upstate) and HDAC3 (Abcam) were used for the second immunoprecipitation, performed overnight at $4^{\circ} \mathrm{C}$. Rabbit IgG $(3 \mu \mathrm{g}$, Santa Cruz Biotechnology Inc.) was used as a negative control for antibody specificity. Elution and cross-link reversal was performed as per manufacturer's instructions (Active Motif). DNA obtained from eluted beads was used for quantitative PCR using Power SYBR Green Master Mix (Applied Biosystems). Primers encompassing the SBE sites of the HGF promoter (above) were used for PCR. Positive binding was defined as antibody binding greater than 10 -fold of the IgG-negative control. $\Delta \mathrm{Ct}$ values were obtained by normalizing IP Ct values to values for chromatin having been immunoprecipitated only once for Smad2 or Smad4 respectively. $\Delta \Delta \mathrm{Ct}$ values were obtained by comparing the $\Delta \mathrm{C} t$ values of Smad4-initial-IP to the Smad2-initial-IP, i.e., Smad4(1st IP)-HDAC3(2nd IP) versus Smad2(1st IP)-HDAC3(2nd IP). Values are expressed as fold change based on $\Delta \Delta \mathrm{Ct}$ values.

$\mathrm{HaCaT}$ keratinocyte culture and siRNA knockdown. HaCaT keratinocytes were cultured in high-glucose DMEM with $10 \%$ FBS and penn-strep antibiotics. Twenty-four hours prior to siRNA transfection, cells were switched to low-glucose DMEM with $0.2 \%$ FBS and penn-strep antibiotics. Cells were transfected with siRNAs for human Smad2, Smad3, or Smad4: Smad2: UUCUCAAGCUCAUCUAACCGUCCUG, Invitrogen (7), or GGAGUGCGCUUAUACUACAtt, Applied Biosystems (Supplemental Figure 5); Smad3: CCUGCUGGAUUGAGCUACACCUGAA, Invitrogen (7), or GUCUACCAGUUGACCCGAAtt, Applied Biosystems (Supplemental Figure 5); Smad4: GGUGAUGUUUGGGUCAGGUGCCUUA, Invitrogen (7), GGACAUUCAAUUCAAACCAtt, Applied Biosystems (Supplemental
Figure 5). XtremeGene siRNA Transfection Reagent (Roche) was used in 6 -well plates at a final concentration of $50 \mathrm{pmol}$ siRNA/ $\mu \mathrm{l}$ in Opti-MEM media (Gibco; Invitrogen). Four hours after transfection, media was switched to high-glucose DMEM. Cells were harvested at 72 hours after siRNA transfection for RNA extraction using QIAshredder and RNeasy kits (QIAGEN). For HaCaT cells treated with TGF- $\beta 1$, a final concentration of $2 \mathrm{ng} / \mathrm{ml} \mathrm{TGF}-\beta 1$ was added to culture medium the last 2 hours prior to harvesting the cells.

Luciferase constructs. Normal C57BL/6 mouse genomic DNA was used to amplify the HGF promoter region using the following set of primers using the Easy-A cloning kit (Promega): FP, CTCCCTCCCTGAAGACTGTG; RP, CTCAGCCCAATCGCTAAGTC. The gel-extracted promoter was ligated to the pGEM T-Easy vector (Promega) as per the manufacturer's instructions. The vector was sequence verified. Using XhoI- and HindIII-labeled forward and reverse primers from above, we amplified the promoter. Using XhoI and HindIII (New England Biosystems) restriction enzymes, we cut the promoter fragment and cloned it into pGL4.26 Luciferase Vector (Promega). A Stratagene QuikChange Kit was used to mutate the SBE from CTGT to TTGA as we previously described (24).

HGF-pGL4.26, pGL4.74 (Promega), and siRNAs were transfected into $50 \%$ confluent HaCaT cells in a 24 -well plate using XtremeGene siRNA Transfection Reagent (Roche). Cells were harvested for luciferase using the Dual Luciferase Assay reagents (Promega) as per the manufacturer's instructions. Luciferase reporter assays were done using the 96 -well plate GloMax dual-injector luminometer (Promega).

Protein extraction and ELISA. Protein extraction was performed by homogenizing tissue in Complete Lysis Buffer M (Roche). Total protein was determined using detergent-compatible Bradford Assay reagents (Bio-Rad). Quantikine ELISA kit for VEGF (R\&D Systems) was used to determine the concentration of VEGF, as per the manufacturer's instructions.

Statistics. Significant differences between the values obtained in each assay on samples from various genotypes were determined using Student's $t$ test and expressed as mean \pm SEM, with the exception of evaluation of human SCCs, where a 2-tailed Fisher's exact test was used. $P$ values considered significant $(P<0.05)$ are denoted in each figure or table.

\section{Acknowledgments}

This work was supported by NIH grants CA87849, CA79998, and DE15953 to X.J. Wang; and R01CA115468 to Q.H. Zhang. $\mathrm{K}$. Hoot is a recipient of NIH training grant HD46420. M. Oka is a recipient of the fellowship from the Uehara Memorial Life Science Foundation, Japan. The authors thank Pamela Garl for proofreading this manuscript.

Received for publication April 12, 2010, and accepted in revised form July 28, 2010.

Address correspondence to: Xiao-Jing Wang, Department of Pathology, Building RC1-N, Room P18-5128, Mail Stop 8104, University of Colorado, Denver, Aurora, Colorado 80045-0508, USA. Phone: 303.724.3001; Fax: 303.724.3712; E-mail: xj.wang@ucdenver.edu.
1. Bierie B, Moses HL. TGFbeta: the molecular Jekyll and Hyde of cancer. Nat Rev Cancer. 2006;6(7):506-520.

2. Massagué J, Seoane J, Wotton D. Smad transcription factors. Genes Dev. 2005;19(23):2783-2810.

3. Massague J, Gomis RR. The logic of TGFbeta signaling. FEBS Lett. 2006;580(12):2811-2820.

4. Wotton D, Lo RS, Lee S, Massague J. A Smad transcriptional corepressor. Cell. 1999;97(1):29-39.

5 . Yu J, et al. Identification of the gene transcription and apoptosis mediated by TGF- $\beta$-Smad $2 / 3-\mathrm{Smad} 4$ sig- naling. J Cell Physiol. 2008;215(2):422-433.

6. Brown KA, Pietenpol JA, Moses HL. A tale of two proteins: differential roles and regulation of Smad 2 and Smad 3 in TGF $\beta$ signaling. $J$ Cell Biochem. 2007;101(1):9-33

7. Hoot KE, et al. Keratinocyte-Specific Smad2 ablation results in increased epithelial-mesenchymal transition during skin cancer formation and progression. J Clin Invest. 2008;118(8):2722-2732.

8 . Bornstein S, et al. Smad4 loss in mice causes sponta- neous head and neck cancer with increased genomic instability and inflammation. J Clin Invest. 2009; 119(11):3408-3419.

9. Tannehill-Gregg SH, Kusewitt DF, Rosol TJ, Weinstein M. The roles of Smad2 and Smad3 in the development of chemically induced skin tumors in mice. Vet Pathol. 2004;41(3):278-282.

10. Yang L, et al. Targeted disruption of Smad 4 in mouse epidermis results in failure of hair follicle cycling and formation of skin tumors. Cancer Res. 2005; 
65(19):8671-8678.

11. Qiao W, Li AG, Owens P, Xu X, Wang XJ, Deng CX. Hair follicle defects and squamous cell carcinoma formation in Smad4 conditional knockout mouse skin. Oncogene. 2006;25(2):207-217.

12. Vijayachandra K, Lee J, Glick AB. Smad3 regulates senescence and malignant conversion in a mouse multistage skin carcinogenesis model. Cancer Res. 2003;63(13):3447-3452.

13. Li AG, Lu SL, Zhang MX, Deng C, Wang XJ. Smad3 knockout mice exhibit a resistance to skin chemical carcinogenesis. Cancer Res. 2004;64(21):7836-7845.

14. Benvenuti S, Comoglio PM. The MET receptor tyrosine kinase in invasion and metastasis. J Cell Physiol. 2007;213(2):316-325.

15. Bussolino F, et al. Hepatocyte growth factor is a potent angiogenic factor which stimulates endothelial cell motility and growth.J Cell Biol. 1992; 119(3):629-641.

16. Chu SH, et al. Hepatocyte growth factor production is stimulated by gangliosides and TGF-beta isoforms in human glioma cells. J Neurooncol. 2007;85(1):33-38.

17. Lewis MP, et al. Tumour-derived TGF-beta1 modulates myofibroblast differentiation and promotes HGF/SF-dependent invasion of squamous carcinoma cells. Br J Cancer. 2004;90(4):822-832.

18. Liu Y, Michalopoulos GK, Zarnegar R. Structural and functional characterization of the mouse hepatocyte growth factor gene promoter. $J$ Biol Chem. 1994;269(6):4152-4160.

19. Plaschke-Schlütter A, Behrens J, Gherardi E, Birchmeier W. Characterization of the scatter factor/hepatocyte growth factor gene promoter. Positive and negative regulatory elements direct gene expression to mesenchymal cells. J Biol Chem. 1995; 270(2):830-836

20. Cheng N, Chytil A, Shyr Y, Joly A, Moses HL. Enhanced hepatocyte growth factor signaling by type II transforming growth factor-beta receptor knockout fibroblasts promotes mammary tumorigenesis. Cancer Res. 2007;67(10):4869-4877.

21. Lebrin F, Deckers M, Bertolino P, Ten Dijke P. TGF-beta receptor function in the endothelium. Cardiovasc Res. 2005;65(3):599-608.

22. Matsumoto K, Nakamura T, Sakai K, Nakamura T. Hepatocyte growth factor and Met in tumor biology and therapeutic approach with NK4. Proteomics. 2008;8(16):3360-3370

23. Lu SL, et al. Loss of transforming growth factro-beta type II receptro preomotes metastatic head-andneck squamous cell carcinoma. Genes Dev. 2006; 20(10):1331-1342.

24. Owens P, Engelking E, Han G, Haeger SM, Wang XJ. Epidermal Smad4 deletion reults in aberrant wound healing. Am J Pathol. 2010;176(1):122-133.

25. Petersen M, et al. Smad2 and Smad3 have opposing roles in breast cacner bone metastasis by differentially affecting tumor angiogenesis. Oncogene. 2010;29(9):1351-1361.

26. Sánchez-Elsner T, Botella LM, Velasco B, Corbí A Attisano L, Bernabéu C. Synergistic cooperation between hypoxia and transforming growth factor-beta pathways on human vascular endothelia growth factor gene expression. J Biol Chem. 2001;276(42):38527-38535.

27. Kobayashi T, et al. Smad3 mediates TGF-beta1 induction of VEGF production in lung fibroblasts. Biochem Biophys Res Commun. 2005;327(2):393-398.

28. Bergers G, Hanahan D. Modes of resistance to anti-angiogenic therapy. Nat Rev Cancer. 2008; 8(8):592-603.

29. Weeks BH, He W, Olson KL, Wang XJ. Inducible expression of transforming growth factor beta 1 in papillomas causes rapid metastasis. Cancer Res. 2001;61(20):7435-7443.

30. Bhowmick NA, et al. TGF-beta signaling in fibroblasts modulates the oncogenic potential of adja- cent epithelia. Science. 2004;303(5659):848-851

31. Sakurai H, Nigam SK. Transforming growth factor-beta selectively inhibits branching morphogenesis but not tubulogenesis. Am J Physiol. 1997; 272(1 pt 2):F139-F146

32. Gherardi E, Gray J, Stoker M, Perryman M, Furlong R. Purification of scatter factor, a fibroblastderived basic protein that modulates epithelial interactions and movement. Proc Natl Acad Sci US A. 1989;86(15):5844-5848.

33. Nakamura T, Teramoto H, Ichihara A. Purification and characterization of a growth factor from rat platelets for mature parenchymal hepatocytes in primary cultures. Proc Natl Acad Sci U S A. 1986; 83(17):6489-6493.

34. Sengupta S, Gherardi E, Sellers LA, Wood JM, Sasisekharan R, Fan TD. Hepatocyte growth factor/scatter factor can induce angiogenesis independently of vascular endothelial growth factor. Arterioscler Thromb Vasc Biol. 2003;23(1):69-75.

35. Ju W, et al. Deletion of Smad2 in mouse liver reveals novel functions in hepatocyte growth and differentiation. Mol Cell Biol. 2006;26(2):654-667.

36. Yang X, Li C, Herrera PL, Deng CX. Generation of Smad4/Dpc4 conditional knockout mice. Genesis. 2002;32(2):80-81.

37. Wang XJ, Greenhalgh DA, Lu XR, Bickenbach JR, Roop DR. TGF alpha and $v$-fos cooperation in transgenic mouse epidermis induces aberrant keratinocyte differentiation and stable, autonomous papillomas. Oncogene. 1995;10(2):279-289.

38. LuSL, et al. Overexpression of transforming growth factor beta 1 in head and neck epithelia results in inflammation, angiogenesis, and epithelial hyperproliferation. Cancer Res. 2004;64(13):4405-4410.

39. Han G, et al. Distinct mechanisms of TGF-beta1mediated epithelial-to-mesenchymal transition and metastasis during skin carcinogenesis. J Clin Invest. 2005;115(7):1714-1723. 